Article

\title{
Polarized Rigid Del Pezzo Surfaces in Low Codimension
}

\author{
Muhammad Imran Qureshi $(D)$ \\ Department of Mathematics, King Fahd University of Petroleum \& Minerals (KFUPM), \\ Dhahran 31261, Saudi Arabia; imran.qureshi@kfupm.edu.sa
}

Received: 11 August 2020; Accepted: 8 September 2020; Published: 11 September 2020

Abstract: We provide explicit graded constructions of orbifold del Pezzo surfaces with rigid orbifold points of type $\left\{k_{i} \times \frac{1}{r_{i}}\left(1, a_{i}\right): 3 \leq r_{i} \leq 10, k_{i} \in \mathbb{Z}_{\geq 0}\right\}$ as well-formed and quasismooth varieties embedded in some weighted projective space. In particular, we present a collection of $147 \mathrm{such}$ surfaces such that their image under their anti-canonical embeddings can be described by using one of the following sets of equations: a single equation, two linearly independent equations, five maximal Pfaffians of $5 \times 5$ skew symmetric matrix, and nine $2 \times 2$ minors of size 3 square matrix. This is a complete classification of such surfaces under certain carefully chosen bounds on the weights of ambient weighted projective spaces and it is largely based on detailed computer-assisted searches by using the computer algebra system MAGMA.

Keywords: orbifold del pezzo surfaces; hypersurfaces; complete intersections; pfaffians; graded ring constructions

\section{Introduction}

A del Pezzo surface is a two dimensional algebraic variety with an ample anti-canonical divisor class. The classification of nonsingular del Pezzo surfaces is well known and there are 10 deformation families of such surfaces: $\mathbb{P}^{1} \times \mathbb{P}^{1}, \mathbb{P}^{2}$ and the blow up of $\mathbb{P}^{2}$ in $d$ general points for $1 \leq d \leq 8$. An orbifold del Pezzo surface $X$ is a del Pezzo surface with at worst isolated orbifold points, classically known as a log del Pezzo surface with cyclic quotient singularities. We describe $X$ to be locally qGorenstein $(q G)$-rigid if it contains only rigid isolated orbifold points, i.e., the orbifold points are rigid under $q G$-deformations. If it admits a $q G$-degeneration to a normal toric del Pezzo surface then it is called a del Pezzo surface of class TG. The Fano index of $X$ is the largest integer $I$ such that $K_{X}=I D$ for an element $D$ in the class group of $X$.

The classification of orbifold del Pezzo surfaces has been an interesting area of research from various points of view, such as the existence of Kahler-Einstein metric [1,2]. Recently, the classification of orbifold del Pezzo surfaces has received much attention, primarily due to the mirror symmetry program for Fano varieties by Coates, Corti et al. [3]. The mirror symmetry for orbifold del Pezzo surface has been formulated in [4] in the form of a conjecture expecting a one to one correspondence between mutation equivalence classes of Fano polygons with the $(q G)$-deformation equivalence classes of locally $q G$-rigid del Pezzo surfaces of class TG. Therefore the construction of rigid orbifold del Pezzo surfaces has important links with the mirror symmetry due to this conjecture. The conjecture has been proved for smooth del Pezzo surfaces by Kasprzyk, Nill and Prince in [5]. Corti and Heuberger [6] gave the classification of locally $q G$-rigid del Pezzo surfaces with $\frac{1}{3}(1,1)$ singular points. The del Pezzo surfaces with a single orbifold point of type $\frac{1}{r}(1,1)$ have been classified by Cavey and Prince [7]. The mutation equivalence classes of Fano polygons with rigid singularities of type

$$
\left\{k_{1} \times \frac{1}{3}(1,1), k_{2} \times \frac{1}{6}(1,1): k_{1}>0, k_{2} \geq 0\right\} \text { and }\left\{k \times \frac{1}{5}(1,1): k>0\right\}
$$


have been computed in [8]. This is equivalent to the classification of del Pezzo surfaces of class TG with the above given baskets; though it may be missing surfaces which do not admit a toric degeneration and having one of the above type of baskets of singularities. By using birational techniques, the classification of orbifold del Pezzo surfaces with basket consisting of a combination of $\frac{1}{3}(1,1)$ and $\frac{1}{4}(1,1)$ orbifold points was given by Miura [9].

In [6] the classification gave a total of 29 deformation families of del Pezzo surfaces with $\frac{1}{3}(1,1)$ orbifold points which were divided into 6 different cascades; one of the cascades was first studied by Reid and Suzuki in [10]. Moreover, good model constructions for all 29 surfaces were presented as complete intersections inside the so called rep-quotient varieties (mainly simplicial toric varieties): A geometric quotient $V / / G$ of a representation $V$ of a complex Lie group $G$. Among those, six of them can be described as a hypersurface in $\mathbb{P}^{3}\left(a_{i}\right)$ or as a complete intersection in $\mathbb{P}^{4}\left(a_{i}\right)$ or as complete intersection in weighted Grassmannian $w \operatorname{Gr}(2,5)$ [11]. This motivated us to classify rigid del Pezzo surfaces with certain basket of singularities which can be described by relatively small sets of equations.

\subsection{Summary of Results}

We classify polarized rigid del Pezzo surfaces, under the bounds chosen in Section 3.2, which contain baskets of orbifold points

$$
\left\{k_{i} \times \frac{1}{r_{i}}\left(1, a_{i}\right): 3 \leq r_{i} \leq 10, k_{i} \geq 0\right\} ;
$$

such that their images under their anti-canonical embedding can be described by one of the following ways.

(i) as a hypersurface, i.e., by a single weighted homogenous equation; $X_{d} \hookrightarrow \mathbb{P}^{3}\left(a_{i}\right)$.

(ii) as a codimension 2 weighted complete intersection, i.e., by 2 weighted homogeneous equations; $X_{d_{1}, d_{2}} \hookrightarrow \mathbb{P}^{4}\left(a_{i}\right)$.

(iii) as a codimension 3 variety described by using five maximal Pfaffians of a $5 \times 5$ skew symmetric matrix;

$$
X_{d_{1}, \ldots, d_{5}} \hookrightarrow \mathbb{P}^{5}\left(a_{i}\right) .
$$

In other words they are weighted complete intersections in weighted Grassmannian ${ } \mathrm{Gr}(2,5)$ or (weighted) projective cone(s) over it [11-13].

(iv) as a codimension 4 variety described by using nine $2 \times 2$ minors of a size 3 square matrix

$$
X_{d_{1}, \ldots, d_{9}} \hookrightarrow \mathbb{P}^{6}\left(a_{i}\right) .
$$

Equivalently, they are weighted complete intersections in some weighted $\mathbb{P}^{2} \times \mathbb{P}^{2}$ variety or (weighted) projective cone(s) over it [14].

We summarize the classification in form of the following theorem.

Theorem 1. Let $X$ be an orbifold del Pezzo surface having at worst a basket

$$
\mathcal{B}=\left\{k_{i} \times \frac{1}{r}(1, a): 3 \leq r \leq 10, k_{i} \geq 0\right\}
$$

of rigid orbifold points and their image $X \hookrightarrow \mathbb{P}\left(a_{i}\right)$ under their anti-canonical embedding can be described as a hypersurface or as a codimension 2 complete intersection or as a weighted complete intersection in $\mathrm{w} G \mathrm{r}(2,5)$ or as a weighted complete intersection of weighted $\mathbb{P}^{2} \times \mathbb{P}^{2}$ variety. Then, subject to Section $3.2, X$ is one of the del Pezzo surfaces listed in Tables A1-A4. In total there are 147 families of such del Pezzo surfaces, divided as follows in each codimension. 


\begin{tabular}{cccc}
\hline Hypersurface & Complete intersection & $4 \times 4$ Pfaffians & $2 \times 2$ Minors \\
\hline 81 & 25 & 21 & 20 \\
\hline
\end{tabular}

We construct these examples by first computing all possible candidate varieties with required basket of orbifold points using an algorithmic approach developed in [15,16], under the bounds given in Section 3.2. In case of codimension 1 and 2, the equations of these varieties are generic weighted homogeneous polynomials of given degrees. In cases of codimension 3 and 4 they are induced from the equations of the corresponding ambient weighted projective variety. We perform a detailed singularity analysis of equations of these candidate varieties to prove the existence or non-existence of given candidate surface. We calculate the $q G$-deformation invariants like the anti-canonical degree $-K_{X}^{2}$ and first plurigenus $h^{0}\left(-K_{X}\right)$ in all cases. We calculate their Euler number and Picard rank in hypersurface case. In complete intersection case, we were able to calculate their Euler number and identify the non-prime examples, i.e., those with the Picard rank greater than 1 by computing their orbifold Euler number.

The computer search used to find these surfaces, based on the algorithm approach of $[15,16]$, is an infinite search. The search is usually performed in the order of increasing sum of the weights $\left(W=\sum a_{i}\right.$ of the ambient weighted projective spaces. In each codimension and for each Fano index I, we provide complete classification of rigid del Pezzo surfaces $X \subset \mathbb{P}\left(a_{i}\right)$ satisfying $W-I \leq N$ where $N \geq 50$ . If the last candidate example for computer search appears for $W-I=q$ then we search for all cases with $N=$ maximum $(50,2 q)$, to minimize the possibility of any further examples. This indeed does not rule out a possibility of further other examples for larger value of $\mathrm{W}$ and I. It is evident that for larger values of $W$ most weights of $\mathbb{P}\left(a_{i}\right)$ will be larger than 10, the highest local index of allowed orbifold points in our classification, consequently the basket of orbifold points will very likely contain orbifold points of local index $r \geq 11$. In cases of hypersurfaces and complete intersections, the classifications of tuples $\left(d_{j} ; \underline{a_{i}}\right)$ which give rise to a quasismooth del Pezzo surfaces can be found in $[17,18]$ where $d_{j}$ denote the degrees of the defining equations and $\underline{a_{i}}$ are weights of the ambient weighted projective space. These classifications of tuples can perhaps be analyzed to give the bound free proof of completeness of our results in codimension 1 and 2 . However, their classification neither contains computation of any of the invariants like $h^{0}\left(-K_{X}\right),-K_{X}^{2}$ and $e(X)$ and nor do they compute the basket of orbifold points lying on those surfaces.

\subsection{Links with Existing Literature}

A part of our search results recovers some existing examples in the literature, though a significant subset of them have not been previoudly described in terms of equations. For example, the classification of Fano polygons (equivalently of rigid del Pezzo surfaces of class TG) with basket of orbifold points (1) is given in [8]. We give descriptions in terms of equations for six of their examples; listed as $14,16,23,85,109$ and 130 in our tables. We also recover the classical smooth del Pezzo surfaces of degrees 1,2,3,4,5, 6 and 8; listed as 3,2,1,82,107,128 and 12 respectively in Tables A1-A4. Moreover, 7 of the 29 examples from [6] also appear in our list with one of them seemingly having a new description as a complete intersection in a $\mathrm{w}\left(\mathbb{P}^{2} \times \mathbb{P}^{2}\right)$ variety, listed as 129 in Table A4. Some examples of Fano index 1 and 2 in codimension 3 and 4 given in Tables A3 and A4 can be found in [19], primarily appearing implicitly as a part of some infinite series of orbifold del Pezzo surfaces.

\section{Background and Notational Conventions}

\subsection{Notation and Conventions}

- We work over the field of complex numbers $\mathbb{C}$.

- All of our varieties are projectively Gorenstein.

- For two orbifold points where $\frac{1}{r}(1, a)=\frac{1}{r}(1, b)$ we choose a presentation $\frac{1}{r}(1, \min (a, b))$. 
- In all the tables, integers appearing as subscripts of $X$ denote the degree of the defining equations of the given variety, where $d^{m}$ means that there are $m$ equations of degree $d$. Similarly, $\mathbb{P}\left(\cdots, a_{i}^{m}, \cdots\right)$ means that there are $m$ weights of degree $a_{i}$.

- We use the same notation for canonical divisor class $K_{X}$ and canonical sheaf $\omega_{X}$, if no confusion can arise. We usually write $K_{X}=\mathcal{O}(k)$ to represent $K_{X}=k D$.

\subsection{Graded Rings and Polarized Varieties}

We call a pair $(X, D)$ a polarized variety if $X$ is a normal projective algebraic variety and $D$ a $\mathbb{Q}$-ample Weil divisor on $X$, i.e., some integer multiple of $D$ is a Cartier divisor. One gets an associated finitely generated graded ring

$$
R(X, D)=\bigoplus_{n \geq 0} H^{0}\left(X, \mathcal{O}_{X}(n D)\right)
$$

It is called a projectively Gorenstein if the ring $R(X, D)$ is a Gorenstein ring. A surjective morphism from a free graded ring $k\left[x_{0}, \ldots, x_{n}\right]$ to $R(X, D)$ gives the embedding

$$
i: X=\operatorname{Proj} R(X, D) \hookrightarrow \mathbb{P}\left(a_{0}, \cdots, a_{n}\right)
$$

where $a_{i}=\operatorname{deg}\left(x_{i}\right)$ and with the divisorial sheaf $\mathcal{O}_{X}(D)$ being isomorphic to $\mathcal{O}_{X}(1)=i^{*} \mathcal{O}_{\mathbb{P}}(1)$. The Hilbert series of a polarized projective variety $(X, D)$ is given by

$$
P_{(X, D)}(t)=\sum_{m \geq 0} h^{0}(X, m D) t^{m},
$$

where $h^{0}(X, m D)=\operatorname{dim} H^{0}\left(X, \mathcal{O}_{X}(m D)\right)$. We usually write $P_{X}(t)$ for the Hilbert series and by the standard Hilbert-Serre theorem [20] (Theorem 11.1), $P_{X}(t)$ has the following compact form

$$
P_{X}(t)=\frac{N(t)}{\prod_{i=0}^{a}\left(1-t^{a_{i}}\right)},
$$

where $N(t)$ is a palindromic polynomial of degree $q$, as $X$ is projectively Gorenstein.

\subsection{Rigid Del Pezzo Surfaces}

Definition 1. An isolated orbifold point $Q$ of type $\frac{1}{r}\left(a_{1}, \ldots, a_{n}\right)$ is the quotient of $\mathbb{A}^{n}$ by the cyclic group $\mu_{r}$,

$$
\epsilon:\left(x_{1}, \ldots, x_{n}\right) \mapsto\left(\epsilon^{a_{1}} x_{1}, \ldots, \epsilon^{a_{n}} x_{n}\right)
$$

such that $\operatorname{GCD}\left(r, a_{i}\right)=1$ for $1 \leq i \leq n, 0<a_{i}<r$, and $\epsilon$ is a primitive generator of $\mu_{r}$.

A del Pezzo surface $X$ is a two dimensional algebraic variety with an ample anti-canonical divisor class $-K_{X}$. If, at worst, $X$ contains isolated orbifold points then we call it an orbifold or a log del Pezzo surface. The Fano index $I$ of $X$ is the largest positive integer $I$ such that $-K_{X}=I \cdot D$ for some divisor $D$ in the divisor class group of $X$. An orbifold del Pezzo surfaces $X \subset \mathbb{P}\left(a_{i}\right)$ of codimension $c$ is well-formed if the singular locus of $X$ consists of at most isolated points. It is quasismooth if the affine cone $\widetilde{X}=\operatorname{Spec} R(X, D) \subset \mathbb{A}^{n+1}$ is smooth outside its vertex $\underline{0}$.

A singularity admitting a $\mathbb{Q}$-Gorenstein smoothing is called a T-singularity [21]. A singularity which is rigid under $\mathbb{Q}$-Gorenstein smoothing is called a rigid or $R$-singularity [22]. The following characterization of a $T$-singularity and $R$-singularity are useful in our context [7].

Definition 2. Let $Q=\frac{1}{r}(a, b)$ be an orbifold point and take $m=\operatorname{GCD}(a+b, r), s=(a+b) / m$ and $k=r / m$ then $Q$ has a form $\frac{1}{m k}(1, m s-1)$. Moreover $Q$ is called a T-singularity if $k \mid m$ [21] and an $\mathrm{R}$-singularity if $m<k$ [22]. 
In the two dimensional case, any orbifold point $\frac{1}{r}(a, b)$ can be represented as $\frac{1}{r}\left(1, a^{\prime}\right)$ by choosing a different primitive generator of the cyclic group $\mu_{r}$ and the following Lemma follows from it.

Lemma 1. Let $Q_{1}=\frac{1}{r}(1, a)$ and $Q_{2}=\frac{1}{r}(1, b)$ be isolated orbifold points. Then $Q_{1}=Q_{2}$ if and only if $a=b$ or $a b \equiv 1 \bmod r$.

By using the fact that each orbifold point on a surface can be written as $\frac{1}{r}(1, a)$ and by applying Lemma 1 on the all possible isolated rigid orbifold points of type $\frac{1}{r}(1, a) ; 3 \leq r \leq 10$, we get to the following Lemma.

Lemma 2. Let $3 \leq r \leq 10$ then any isolated rigid orbifold point $\frac{1}{r}(a, b)$ is equivalent to one of the following.

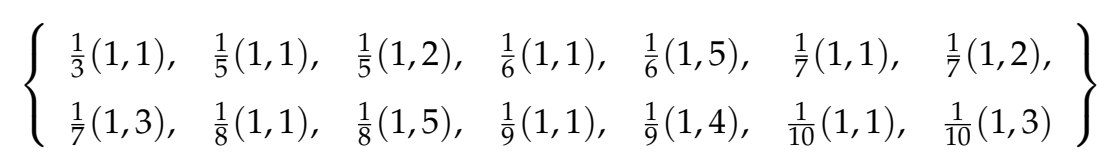

\subsection{Ambient Varieties}

In this section we briefly recall the definition of weighted Grassmannian $w \operatorname{Gr}(2,5)$ and $w\left(\mathbb{P}^{2} \times \mathbb{P}^{2}\right)$ which we use, apart from weighted projective spaces, as rep-quotient varieties for the construction of our rigid orbifold del Pezzo surfaces; following the notion introduced in [6].

\subsubsection{Weighted Grassmannian wGr $(2,5)$}

This part is wholly based on material from ([11], Section 2$)$. Let $w:=\left(w_{1}, \cdots, w_{5}\right)$ be a tuple of all integers or all half integers such that

$$
w_{i}+w_{j}>0,1 \leq i<j \leq 5,
$$

Then the quotient of the affine cone over Grassmannian minus the origin $\widetilde{\operatorname{Gr}(2,5)} \backslash\{\underline{0}\}$ by $\mathbb{C}^{\times}$given by:

$$
\epsilon: x_{i j} \mapsto \epsilon^{w_{i}+w_{j}} x_{i j}
$$

is called weighted Grassmannians $w \mathrm{Gr}(2,5)$ where $x_{i j}$ are Plücker coordinates of the embedding $\operatorname{Gr}(2,5) \hookrightarrow \mathbb{P}\left(\wedge^{2} \mathbb{C}^{5}\right)$. Therefore we get the embedding

$$
\mathrm{wGr}(2,5) \hookrightarrow \mathbb{P}\left(a_{i j}: 1 \leq i<j \leq 5, a_{i j}=w_{i}+w_{j}\right) .
$$

The image of $\operatorname{Gr}(2,5)$ and $w \operatorname{Gr}(2,5)$ under the Plücker embedding is defined by five $4 \times 4$ Pfaffians of the $5 \times 5$ skew symmetric matrix

$$
\left(\begin{array}{llll}
x_{12} & x_{13} & x_{14} & x_{15} \\
& x_{23} & x_{24} & x_{25} \\
& & x_{34} & x_{35} \\
& & & x_{45}
\end{array}\right)
$$

where we only write down the upper triangular part. Explicitly, the defining equations are:

$$
\mathrm{Pf}_{i}=x_{j k} x_{l m}-x_{j l} x_{k m}+x_{j m} x_{l m}
$$

where $1 \leq j<k<l<m \leq 5$ are four integers and $i$ makes up the fifth one in $\{1,2,3,4,5\}$. In examples we usually write down the corresponding matrix of weights, replacing $x_{i j}$ with $a_{i j}$ to represent the given $\mathrm{wGr}(2,5)$. 
If ${ }_{w} \mathrm{Gr}(2,5)$ is wellformed then the orbifold canonical divisor class is

$$
K_{\mathrm{wGr}(2,5)}=\left(-\frac{1}{2} \sum_{1 \leq i<j \leq 5} a_{i j}\right) D,
$$

for a divisor $D$ in the class group of ${ }_{\mathrm{w}} \mathrm{Gr}(2,5)$.

\subsubsection{Weighted $\mathbb{P}^{2} \times \mathbb{P}^{2}$}

This section recalls the definition of weighted $\mathbb{P}^{2} \times \mathbb{P}^{2}$ from $[14,23]$. Let $b=\left(b_{1}, b_{2}, b_{3}\right)$ and $c=\left(c_{1}, c_{2}, c_{3}\right)$ be two integer or half integer vectors satisfying

$$
b_{1}+c_{1}>0, \quad b_{i} \leq b_{j} \text { and } \quad c_{i} \leq c_{j} \text { for } 1 \leq i \leq j \leq 3
$$

and $\Sigma_{P}$ denotes the Segre embedding $\mathbb{P}^{2} \times \mathbb{P}^{2} \hookrightarrow \mathbb{P}^{8}\left(x_{i j}\right)$. If $\widetilde{\Sigma_{P}}$ is the affine of this Segre embedding,

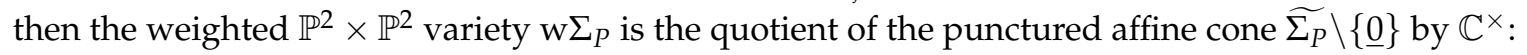

$$
\epsilon: x_{i j} \mapsto \epsilon^{b_{i}+c_{j}} x_{i j}, 1 \leq i, j \leq 3
$$

Thus for a choice of $b, c$, written together as a single input parameter $p=\left(b_{1}, b_{2}, b_{3} ; c_{1}, c_{2}, c_{3}\right)$, we get the embedding

$$
\mathrm{w} \Sigma_{P} \hookrightarrow \mathbb{P}^{8}\left(a_{i j}: a_{i j}=b_{i}+c_{j} ; 1 \leq i, j \leq 3\right) .
$$

The equations are defined by $2 \times 2$ minors of a size 3 square matrix which we usually refer to as the weight matrix and write it as

$$
\left(\begin{array}{lll}
a_{11} & a_{12} & a_{13} \\
a_{21} & a_{22} & a_{23} \\
a_{31} & a_{32} & a_{33}
\end{array}\right) \text { where } a_{i j}=b_{i}+c_{j} ; 1 \leq i, j \leq 3 .
$$

If $\mathrm{w} \Sigma_{P}$ is wellformed then the canonical divisor class is given by

$$
K_{\mathrm{w} \Sigma_{P}}=\left(-\sum_{i=j} a_{i j}\right) D,
$$

for a divisor $D$ in the class group of ${ }_{\mathrm{w}} \Sigma_{P}$.

\section{Computational Steps of The Proof}

In this section we provide details of various steps of our calculations which together provide the proof of Theorem 1. In summary, for each codimension and Fano index, we first search for the list of candidate varieties using the algorithmic approach of $[15,16]$. The candidate lists comes with a suggestive basket(s) of orbifold points and invariants. Then we perform theoretical analysis of each candidate to establish the existence or non-existence of candidate surfaces with given basket and invariants.

\subsection{Algorithm}

We briefly recall the algorithm from [16] which we used to compute the candidate lists of examples. The key part of it is based on the orbifold Riemann-Roch formula of Bukcley, Reid and Zhou [24] which provides a decomposition of the Hilbert series of $X$ into a smooth part and a singular part. It roughly states that if $X$ is an algebraic variety with basket $\mathcal{B}=\left\{k_{i} \times Q_{i}: m_{i} \in \mathbb{Z}_{>0}\right\}$ of isolated orbifold points then its Hilbert series has a decomposition into a smooth part $P_{\mathrm{sm}}(t)$ and orbifold part $\sum k_{i} P_{Q_{i}}(t)$;

$$
P_{X}(t)=P_{\mathrm{sm}}(t)+\sum k_{i} P_{Q_{i}}(t) .
$$


The algorithm searches for all orbifolds of fixed dimension $n$ having fixed orbifold canonical class $K_{X}=\mathcal{O}(k)$ in a given ambient rep-quotient variety. Indeed, if $X$ is a Fano variety of index $I$ then $k=-I$. The algorithm has the following steps.

(i) Compute the Hilbert series and orbifold canonical class of ambient rep-quotient variety.

(ii) Find all possible embeddings of $n$-folds $X$ with $\omega_{X}=\mathcal{O}(k)$ by applying the adjunction formula.

(iii) For each possible $n$-fold embedding of $X$, compute the Hilbert series $P_{X}(t)$ and the smooth term $P_{\mathrm{sm}}(t)$.

(iv) Compute the list of all possible $n$-fold isolated orbifold points from the ambient weighted projective space containing $X$.

(v) For each subset of the list of possible orbifold points determine the multiplicities $k_{i}$ given in Equation (8) of the orbifold terms $P_{Q_{i}}(t)$.

(vi) If $k_{i} \geq 0$ then $X$ is a candidate $n$-fold with suggested basket of isolated orbifold points.

\subsection{Bounds on Search Parameters}

We perform our search in the order of increasing sum of the weights on the ambient weight projective space $\mathbb{P}\left(a_{0}, \ldots, a_{n}\right)$ containing $X$. The search is theoretically unbounded in each codimension in two directions: there is no bound on the sum of weights $W=\sum a_{i}$ of the ambient weighted projective space containing $X$ and the Fano index $I$ is also unbounded.

In each codimension, we at least search for polarized rigid del Pezzo surfaces $X \hookrightarrow \mathbb{P}\left(a_{i}\right)$ such that

$$
W-I \leq 50, \text { for } 1 \leq I \leq 10
$$

If the last candidate example is found for the adjunction number $q=W-I$ of the Hilbert numerator $N(t)$, then we further search for all possible cases such that

$$
W-I \leq N \text { where } N=\operatorname{maximum}(2 q, 50),
$$

to absolutely minimize the possibility of any missing examples. Similarly, in each codimension if we find the last example in search domain $W-I \leq 50$ for index $I>5$ then we search for examples up to index $2 I$. For example, in the hypersurface case the maximum value of $I$ across all candidates was 8 , so we searched until index 16 in this case. Similarly, for index 2 hypersurfaces we got the last candidate when $W-2=36$ so we searched for all cases with $W-2 \leq 72$. Further details in each case can be found in Table 1.

Table 1. The following table summarises the number of surfaces we obtained for each Fano index $I$ in each codimension and exact search domain in each case. First column contains the codimension of each surface and the rest of the columns contain a pair of numbers. First number is the number of examples of given index and the second one gives the maximum value of $q=W_{\max }-I$ for which the last candidate surface was found; the classification is complete until $N=$ maximum $(50,2 q)$. The entries with no second number means that no examples were found for $q \leq 50$.

\begin{tabular}{llllllllll}
\hline \multirow{2}{*}{ Codimension } & \multicolumn{10}{c}{ Fano Index, $(\boldsymbol{q})$} \\
\cline { 2 - 12 } & $\mathbf{1}$ & $\mathbf{2}$ & $\mathbf{3}$ & $\mathbf{4}$ & $\mathbf{5}$ & $\mathbf{6}$ & $\mathbf{7}$ & $\mathbf{8}$ & $\mathbf{9 - 1 6}$ \\
\hline 1 & $11(28)$ & $44(36)$ & $6(15)$ & $\mathbf{6 ( 2 1 )}$ & $\mathbf{6 ( 2 1 )}$ & $\mathbf{2 ( 1 6 )}$ & $\mathbf{2 ( 1 7 )}$ & $4(15)$ & 0 \\
\hline 2 & $15(22)$ & $8(29)$ & $1(26)$ & $1(22)$ & 0 & 0 & 0 & 0 & \\
\hline 3 & $12(33)$ & $7(43)$ & $1(19)$ & $1(26)$ & 0 & 0 & 0 & 0 & \\
\hline 4 & $12(42)$ & $6(48)$ & 0 & 0 & $1(30)$ & 0 & 0 & $1(42)$ & 0 \\
\hline
\end{tabular}

\subsection{Computing Invariants}

We describe how we calculate each of the following $q G$-deformation invariants appearing in Tables A1-A4. 
(i) First plurigenus $h^{0}\left(-K_{X}\right)$ : If it is equal to zero then we can easily conclude that $X$ does not admit a $q G$-deformation to a toric variety and such surfaces are not of class TG. We compute it as the coefficient of $t^{I}$ in the Hilbert series (2) where $I$ is the Fano index of $X$.

(ii) Intersection number $-K_{X}^{2}$ : It can be defined as an anti-canonical degree of $X$ which we calculate from the Hilbert series $P_{X}(t)$ of $X$. In a surface case

$$
P_{X}(t)=\frac{H(t)}{(1-t)^{3}}
$$

where $H(t)$ is a rational function with only positive coefficients. Then for a generic divisor $D$ in the class group, we have $D^{2}=H(1)$. Consequently for an orbifold del Pezzo surface of index $I$, we have $-K_{X}^{2}=I^{2} D^{2}$.

(iii) Euler Characteristics $e(X)$ : We were able to compute the Euler characteristics of $X$ in hypersurface and complete intersection cases by using Blache's formula ([25], 2.11-14);

$$
e(X)=e_{\mathrm{orb}}(X)+\sum_{r(Q) \in \mathcal{B}} \frac{r-1}{r}
$$

where $r$ is the local index of each orbifold point. It was applied in the Appendix of [26] to illustrate the computation for a hypersurface. The formula has natural generalization to the cases of complete intersections

$$
X_{d_{1}, \ldots, d_{k}} \subset \mathbb{P}\left(a_{0}, \ldots, a_{n}\right)
$$

in higher codimension. We can computer $e_{\mathrm{orb}}(X)$ as:

$$
e_{\mathrm{orb}}(X)=\text { coefficient of } t^{n-k} \text { in the series expansion of }\left(\frac{\prod\left(1+a_{i} t\right)}{\prod\left(1+d_{i} t\right)} \operatorname{deg}(X)\right) \text {. }
$$

(iv) Picard rank $\rho(x)$ : We were able to calculate it explicitly when $X$ is a hypersurface in $\mathbb{P}^{3}\left(a_{i}\right)$ by using ([27], Sec. 4.4.1). Given a hypersurface

$$
X_{d} \hookrightarrow \mathbb{P}\left(a_{0}, a_{1}, a_{2}, a_{3}\right),
$$

let

$$
l=\text { coefficient of } t^{2 d-\sum a_{i}} \text { in the series expansion of }\left(\prod \frac{t^{d-a_{i}}-1}{t^{a_{i}}-1}\right),
$$

then $\rho(X)=l+1$. In cases of complete intersection examples we were able to identify those examples which are not prime, i.e., the Picard rank greater than 1. From [28], we know that if the Picard rank of a log del Pezzo surface is 1 then $0<e_{\text {orb }}(X) \leq 3$. Therefore, for each codimension 2, we complete the intersection in Table A2, we list $e_{\mathrm{orb}}(X)$ and those with $e_{\text {orb }}(X)>3$ have Picard rank greater than 1.

\subsection{Theoretical Singularity Analysis}

The last step of the calculation is the theoretical singularity analysis of each candidate orbifold. We prove that the general member $X$ in each family is wellformed and quasismooth. We first compute the dimensions of intersection of all orbifold strata with $X$ to establish that $X$ is wellformed. This should be less than or equal to zero for a surface to be wellformed, i.e., it does not contain any singular lines.

The next step is to show that $X$ is quasismooth. This is not so difficult when $X$ is a hypersurface or complete intersection: one can use the criteria given in ([29], Sec. 8). In cases of codimension 3 and 4 examples, we consider $X$ as complete intersections in $\operatorname{wGr}(2,5)$ or in the Segre embedding of weighted $\mathbb{P}^{2} \times \mathbb{P}^{2}$ or in some projective cone(s) over either of those ambient varieties. So $X$ may not only have 
singularities from the ambient weighted projective but it may also contain singularities on the base loci of linear systems of the intersecting weighted homogeneous forms. In such cases we mostly prove the quasismoothness on the base locus by using computer algebra system MAGMA [30]. We write down explicit equations for $X$ over the rational numbers and show that it is smooth, see ([19], Sec. 2.3) for more details. To prove quasismoothness on an orbifold point $Q$ of type $\frac{1}{r}(a, b)$, which is mostly a coordinate point corresponding to some variables $x_{i}$ with $\operatorname{deg}\left(x_{i}\right)=r$, we proceed as follows. If $c$ is the codimension of $X$ then we find $c$ tangent variables $x_{m}$ [31], i.e., we find $c$ polynomials having a monomial of type $x_{i}^{l} x_{m}$. We can locally remove these variables by using the implicit function theorem. Moreover, if two other variables have weights $a$ and $b$ modulo $r$ then $Q$ is a quasismooth point of type $\frac{1}{r}(a, b)$.

\section{Sample Calculations}

In this section we provide sample calculations of examples given in Tables A1-A4.

Example 1. \#81 Consider the weighted projective space $\mathbb{P}(1,5,7,10)$ with variables $x, y, z$ and $w$ respectively, then the canonical class $K_{\mathbb{P}}=\mathcal{O}(-23)$. The generic weighted homogenous polynomial of degree 15,

$$
f_{15}=k_{1} x^{15}+k_{2} y^{3}+k_{3} y w+k_{4} x z^{2}+\cdots, \quad k_{i} \in \mathbb{C} ;
$$

defines a del Pezzo surface $X_{15} \hookrightarrow \mathbb{P}(x, y, z, w)$ of Fano index 8 , i.e., $K_{X}=\mathcal{O}(-8)$. The polynomial $f_{15}$ does not contain monomials of pure power in $w$ and $z$ so $X$ contains the orbifold points $p_{1}=(0,0,0,1)$ and $p_{2}=(0,0,1,0)$. By applying the implicit function theorem we can remove the variable $y$ near the point $p_{1}$ by using the monomial $y w$ and $x, z$ are local variables near this point. Therefore $X$ contains an orbifold point of type $\frac{1}{10}(1,7)=\frac{1}{10}(1,3)\left(\right.$ Lemma 1). Similarly, near $p_{2}$ the local variables are $y$ and $w$, so we get an orbifold point of type

$$
\frac{1}{7}(5,10)=\frac{1}{7}(3,5)=\frac{1}{7}(1,4)=\frac{1}{7}(1,2) .
$$

The coordinate point of weight 5 does not lie on $X$ but one dimensional singular stratum $\mathbb{P}^{1}(y, w)$ intersects with X non-trivially and by ([29], Lemma 9.4) the intersection is in two points. One of them is $p_{1}$ and the other can be taken as $p_{3}=(0,1,0,0)$ which corresponds to weight 5 variable. By using the above arguments we can show that it is a singular point of type $\frac{1}{5}(1,2)$. Thus X contains exactly the same basket of singularities as given by the computer search and it is a wellformed and quasismooth rigid del Pezzo surface of Fano index 8. Moreover, the vector space

$$
H^{0}\left(X,-K_{X}\right)=H^{0}(X, 8 D)=<x^{8}, x^{3} y, x z>\text {, }
$$

so $h^{0}\left(-K_{X}\right)=3$.

Example 2. \#126 Consider the weighted Grassmannian wGr(2,5)

$$
\mathrm{wGr}(2,5) \hookrightarrow \mathbb{P}\left(1^{2}, 3^{3}, 5^{4}, 7\right) \text { with weight matrix }\left(\begin{array}{rrrr}
1 & 1 & 3 & 3 \\
& 3 & 5 & 5 \\
& & 5 & 5 \\
& & & 7
\end{array}\right) \text {, }
$$

Then by Equation (5) the canonical divisor class $K_{\mathrm{w} G r(2,5)}=\mathcal{O}(-19)$. The weighted complete intersection of $\mathrm{WGr}(2,5)$ with two forms of degree 3 and two forms of degree 5 ;

$$
X=w \operatorname{wr}(2,5) \cap\left(f_{3}\right) \cap\left(g_{3}\right) \cap\left(f_{5}\right) \cap\left(g_{5}\right) \hookrightarrow \mathbb{P}_{\left(x_{1}, x_{2}, y_{1}, z_{1}, z_{2}, w_{1}\right)}\left(1^{2}, 3,5^{2}, 7\right)
$$


is a del Pezzo surface with $K_{X}=\mathcal{O}(-19+(3+3+5+5))=\mathcal{O}(-3)$. We can take $X$ to be defined by the maximal Pfaffians of

$$
\left(\begin{array}{cccc}
x_{1} & x_{2} & f_{3} & g_{3} \\
& y_{1} & f_{5} & g_{5} \\
& & z_{1} & z_{2} \\
& & & w_{1}
\end{array}\right),
$$

where $f_{3}, g_{3}, f_{5}$ and $g_{5}$ are general weighted homogeneous forms in given variables and they remove the variables of the corresponding degrees from the ambient $\mathrm{w} G \mathrm{r}(2,5)$. The coordinate point corresponding to $w_{1}$ lies on $X$. From the equations we have $x_{1}, x_{2}$ and $y_{1}$ as tangent variables and $z_{1}, z_{2}$ as local variables. Therefore it is an orbifold point of type $\frac{1}{7}(5,5)=\frac{1}{7}(1,1)$. The locus $X \cap \mathbb{P}(5,5)$ is locally a quadric in $\mathbb{P}^{1}$ which defines two points. By similar application of implicit function theorem we can show that each is an orbifold point of type $\frac{1}{5}(1,2)$. The restriction of $X$ to weight 3 locus is an empty set, so $X$ contains no further orbifold points. To show the quasismoothness on the base locus we use the computer algebra and write down equations for $X$. For example, if we choose

$$
\begin{array}{cc}
f_{3}=3 x_{1}^{3}+3 x_{2}^{3}, & f_{5}=x_{2}^{5}+x_{1}^{2} y_{1}+x_{2}^{2} y_{1}+z_{1}+z_{2}, \\
g_{3}=x_{2}^{3}+y_{1}, & g_{5}=x_{1}^{5}+2 x_{1}^{2} y_{1}+3 x_{2}^{2} y_{1}+3 z_{2}
\end{array}
$$

then the Pfaffians of (11) gives a quasismooth surface. Thus X is an orbifold del Pezzo surface of Fano index 3 with singular points; $2 \times \frac{1}{5}(1,2)$ and $\frac{1}{7}(1,1)$.

As we mentioned in Section 3.4 that we prove the existence of given orbifold del Pezzo surface by theoretical singularity analysis. Then only those which are quasismooth, wellformed and having correct basket of singularities appear in tables of examples. There are in total 8 candidate examples which fails to be quasismooth and we discuss one of them below in detail. No candidate example fails for not being wellformed.

Example 3. (Non working candidate) A computer search also gives a candidate complete intersection orbifold del Pezzo surface of Fano index 2 given by

$$
X_{6,30} \hookrightarrow \mathbb{P}_{(x, y, z, t, u)}(1,3,9,10,15) .
$$

Then $F_{6}=f(x, y)$ (since other variables have weight higher than 6) and

$$
F_{30}=x^{30}+x^{27} y+y z^{3}+\cdots
$$

are the defining equations of $X$. The coordinate point $p=(0,0,1,0,0)$ lies on $X$ as no pure power of $z$ appear in $F_{30}$. Now we can not find two tangent variables to $z$ in the equations of $X$ which implies that the rank of the Jacobian matrix of $X$ at $p$ is equal to 1 which is less than its codimension, so $X$ is not quasismooth at $p$. Thus, $X$ is a del Pezzo surface which is not quasismooth and does not appear in the following tables.

Concluding Remark: One can use this approach to construct and classify orbifold del Pezzo surfaces with any quotient singularity in a given fixed format, under certain bounds. Moreover, we can also construct examples with rigid orbifold points of type $\frac{1}{r}(1, a)$ for $r \geq 11$ but as the weights higher the computer search output becomes slower due to the nature of algorithm. Therefore, we restrict ourself to the cases with $r \leq 10$.

Funding: This research was funded by the Deanship of Scientific Research, King Fahd University of Petroleum and Minerals via a grant number SB. 191029.

Acknowledgments: I would like to thank Erik Paemurru for pointing me to [27] for computations of Picard rank in the hypersurface case. I am grateful to an anonymous referee for their feedback which improved the exposition of the paper significantly.

Conflicts of Interest: The author declares no conflict of interest. 


\section{Appendix A. Table of Examples}

\section{Notations in Tables}

- The column $X$ represents a del Pezzo surface and the corresponding weighted projective space containing $X$; the subscripts give the equation degrees of $X$. The column $I$ lists the Fano index of $X$.

- The next two columns contain the anti-canonical degree $-K_{X}^{2}$ and the first plurigenus $h^{0}\left(-K_{X}\right)$. If $h^{0}\left(-K_{X}\right)=0$ the $X$ is not of class $T G$.

- $\quad e(X)$ denotes the topological Euler characteristics of $X, \rho(X)$ is the rank of Picard group of $X$, and $e_{\mathrm{orb}}(X)$ denotes the orbifold Euler number of $X . \rho(X)$ is only listed in Table A1 of hypersurfaces and $e_{\mathrm{orb}}(X)$ only in Table $\mathrm{A} 2$ of complete intersections, as discussed in Section 3.3.

- $\quad$ The column $\mathcal{B}$ represents the basket of singular points of $X$.

- In Tables A3 and A4, the last column represents the matrix of weights, which provides weights of ambient weighted projective space containing wGr $(2,5)$ or weighted $\mathbb{P}^{2} \times \mathbb{P}^{2}$ variety.

- We provide references to those examples which appeared in $[6,8]$, primarily in a toric setting.

Table A1. Hypersurfaces in $w \mathbb{P}^{3}$.

\begin{tabular}{llcccccc}
\hline S.No & \multicolumn{1}{c}{$\boldsymbol{X}$} & $\boldsymbol{I}$ & $-\boldsymbol{K}_{\boldsymbol{X}}^{2}$ & $\boldsymbol{h}^{\mathbf{0}}\left(-\boldsymbol{K}_{X}\right)$ & $\boldsymbol{e}(\boldsymbol{X})$ & $\boldsymbol{\rho}(\boldsymbol{X})$ & Basket $\mathcal{B}$ \\
\hline 1 & $X_{3} \subset \mathbb{P}\left(1^{4}\right)$ & 1 & 3 & 4 & 9 & 7 & \\
2 & $X_{4} \subset \mathbb{P}\left(1^{3}, 2\right)$ & 1 & 2 & 3 & 10 & 8 & \\
3 & $X_{6} \subset \mathbb{P}\left(1^{2}, 2,3\right)$ & 1 & 1 & 2 & 11 & 9 & \\
4 & $X_{10} \subset \mathbb{P}(1,2,3,5)$ & 1 & $1 / 3$ & 1 & 11 & 9 & $\frac{1}{3}(1,1)[6]$ \\
5 & $X_{12} \subset \mathbb{P}\left(2,3^{2}, 5\right)$ & 1 & $2 / 15$ & 0 & 10 & 8 & $4 \times \frac{1}{3}(1,1), \frac{1}{5}(1,1)$ \\
6 & $X_{15} \subset \mathbb{P}(1,3,5,7)$ & 1 & $1 / 7$ & 1 & 11 & 9 & $\frac{1}{7}(1,2)$ \\
7 & $X_{15} \subset \mathbb{P}\left(3^{2}, 5^{2}\right)$ & 1 & $1 / 15$ & 0 & 11 & 9 & $5 \times \frac{1}{3}(1,1), 3 \times \frac{1}{5}(1,1)$ \\
8 & $X_{16} \subset \mathbb{P}(1,3,5,8)$ & 1 & $2 / 15$ & 1 & 12 & 10 & $\frac{1}{3}(1,1), \frac{1}{5}(1,1)$ \\
9 & $X_{18} \subset \mathbb{P}(2,3,5,9)$ & 1 & $1 / 15$ & 0 & 9 & 7 & $2 \times \frac{1}{3}(1,1), \frac{1}{5}(1,2)$ \\
10 & $X_{20} \subset \mathbb{P}\left(2,5^{2}, 9\right)$ & 1 & $2 / 45$ & 0 & 10 & 8 & $4 \times \frac{1}{5}(1,2), \frac{1}{9}(1,1)$ \\
11 & $X_{28} \subset \mathbb{P}(3,5,7,14)$ & 1 & $2 / 105$ & 0 & 8 & 6 & $\frac{1}{3}(1,1), \frac{1}{5}(1,2), 2 \times \frac{1}{7}(1,2)$ \\
\hline 12 & $X_{2} \subset \mathbb{P}\left(1^{4}\right)$ & 2 & 8 & 9 & 4 & 2 & \\
13 & $X_{4} \subset \mathbb{P}\left(1^{3}, 3\right)$ & 2 & $16 / 3$ & 6 & 6 & 4 & $\frac{1}{3}(1,1)[6]$ \\
14 & $X_{6} \subset \mathbb{P}\left(1^{3}, 5\right)$ & 2 & $24 / 5$ & 6 & 8 & 6 & $\frac{1}{5}(1,1)[8]$ \\
15 & $X_{6} \subset \mathbb{P}\left(1^{2}, 3^{2}\right)$ & 2 & $8 / 3$ & 3 & 8 & 6 & $2 \times \frac{1}{3}(1,1)[6]$ \\
16 & $X_{7} \subset \mathbb{P}\left(1^{3}, 6\right)$ & 2 & $14 / 3$ & 6 & 9 & 7 & $\frac{1}{6}(1,1)[8]$ \\
17 & $X_{8} \subset \mathbb{P}\left(1^{2}, 3,5\right)$ & 2 & $32 / 15$ & 3 & 10 & 8 & $\frac{1}{3}(1,1), \frac{1}{5}(1,1)$ \\
18 & $X_{8} \subset \mathbb{P}\left(1^{3}, 7\right)$ & 2 & $32 / 7$ & 6 & 10 & 8 & $\frac{1}{7}(1,1)$ \\
19 & $X_{9} \subset \mathbb{P}\left(1^{2}, 3,6\right)$ & 2 & 2 & 3 & 11 & 9 & $\frac{1}{3}(1,1), \frac{1}{6}(1,1)[8]$ \\
20 & $X_{9} \subset \mathbb{P}\left(1^{3}, 8\right)$ & 2 & $9 / 2$ & 6 & 11 & 9 & $\frac{1}{8}(1,1)$ \\
21 & $X_{10} \subset \mathbb{P}\left(1^{2}, 3,7\right)$ & 2 & $40 / 21$ & 3 & 12 & 10 & $\frac{1}{3}(1,1), \frac{1}{7}(1,1)$ \\
22 & $X_{10} \subset \mathbb{P}\left(1^{3}, 9\right)$ & 2 & $40 / 9$ & 6 & 12 & 10 & $\frac{1}{9}(1,1)$ \\
23 & $X_{10} \subset \mathbb{P}\left(1^{2}, 5^{2}\right)$ & 2 & $8 / 5$ & 3 & 12 & 10 & $2 \times \frac{1}{5}(1,1)[8]$ \\
24 & $X_{11} \subset \mathbb{P}\left(1^{3}, 10\right)$ & 2 & $22 / 5$ & 6 & 13 & 11 & $\frac{1}{10}(1,1)$ \\
25 & $X_{11} \subset \mathbb{P}\left(1^{2}, 5,6\right)$ & 2 & $22 / 15$ & 3 & 13 & 11 & $\frac{1}{5}(1,1), \frac{1}{6}(1,1)$ \\
26 & $X_{11} \subset \mathbb{P}\left(1^{2}, 3,8\right)$ & 2 & $11 / 6$ & 3 & 13 & 11 & $\frac{1}{3}(1,1), \frac{1}{8}(1,1)$ \\
27 & $X_{12} \subset \mathbb{P}\left(1^{2}, 5,7\right)$ & 2 & $48 / 35$ & 3 & 14 & 12 & $\frac{1}{5}(1,1), \frac{1}{7}(1,1)$ \\
\hline & & & & & & & \\
\hline
\end{tabular}


Table A1. Cont.

\begin{tabular}{|c|c|c|c|c|c|c|c|}
\hline S.No & $X$ & $I$ & $-K_{X}^{2}$ & $h^{0}\left(-K_{X}\right)$ & $e(X)$ & $\rho(X)$ & Basket $\mathcal{B}$ \\
\hline 28 & $X_{12} \subset \mathbb{P}\left(1^{2}, 6^{2}\right)$ & 2 & $4 / 3$ & 3 & 14 & 12 & $2 \times \frac{1}{6}(1,1)[8]$ \\
\hline 29 & $X_{12} \subset \mathbb{P}\left(1^{2}, 3,9\right)$ & 2 & $16 / 9$ & 3 & 14 & 12 & $\frac{1}{3}(1,1), \frac{1}{9}(1,1)$ \\
\hline 30 & $X_{13} \subset \mathbb{P}\left(1^{2}, 5,8\right)$ & 2 & $13 / 10$ & 3 & 15 & 13 & $\frac{1}{5}(1,1), \frac{1}{8}(1,1)$ \\
\hline 31 & $X_{13} \subset \mathbb{P}\left(1^{2}, 3,10\right)$ & 2 & $26 / 15$ & 3 & 15 & 13 & $\frac{1}{3}(1,1), \frac{1}{10}(1,1)$ \\
\hline 32 & $X_{13} \subset \mathbb{P}\left(1^{2}, 6,7\right)$ & 2 & $26 / 21$ & 3 & 15 & 13 & $\frac{1}{6}(1,1), \frac{1}{7}(1,1)$ \\
\hline 33 & $X_{14} \subset \mathbb{P}\left(1^{2}, 6,8\right)$ & 2 & $7 / 6$ & 3 & 16 & 14 & $\frac{1}{6}(1,1), \frac{1}{8}(1,1)$ \\
\hline 34 & $X_{14} \subset \mathbb{P}\left(1^{2}, 7^{2}\right)$ & 2 & $8 / 7$ & 3 & 16 & 14 & $2 \times \frac{1}{7}(1,1)$ \\
\hline 35 & $X_{14} \subset \mathbb{P}\left(1^{2}, 5,9\right)$ & 2 & $56 / 45$ & 3 & 16 & 14 & $\frac{1}{5}(1,1), \frac{1}{9}(1,1)$ \\
\hline 36 & $X_{15} \subset \mathbb{P}\left(1^{2}, 6,9\right)$ & 2 & $10 / 9$ & 3 & 17 & 15 & $\frac{1}{6}(1,1), \frac{1}{9}(1,1)$ \\
\hline 37 & $X_{15} \subset \mathbb{P}\left(1^{2}, 5,10\right)$ & 2 & $6 / 5$ & 3 & 17 & 15 & $\frac{1}{5}(1,1), \frac{1}{10}(1,1)$ \\
\hline 38 & $X_{15} \subset \mathbb{P}(1,3,6,7)$ & 2 & $10 / 21$ & 1 & 11 & 9 & $2 \times \frac{1}{3}(1,1), \frac{1}{6}(1,1), \frac{1}{7}(1,2)$ \\
\hline 39 & $X_{15} \subset \mathbb{P}\left(1^{2}, 7,8\right)$ & 2 & $15 / 14$ & 3 & 17 & 15 & $\frac{1}{7}(1,1), \frac{1}{8}(1,1)$ \\
\hline 40 & $X_{16} \subset \mathbb{P}\left(1^{2}, 6,10\right)$ & 2 & $16 / 15$ & 3 & 18 & 16 & $\frac{1}{6}(1,1), \frac{1}{10}(1,1)$ \\
\hline 41 & $X_{16} \subset \mathbb{P}\left(1^{2}, 7,9\right)$ & 2 & $64 / 63$ & 3 & 18 & 16 & $\frac{1}{7}(1,1), \frac{1}{9}(1,1)$ \\
\hline 42 & $X_{16} \subset \mathbb{P}\left(1^{2}, 8^{2}\right)$ & 2 & 1 & 3 & 18 & 16 & $2 \times \frac{1}{8}(1,1)$ \\
\hline 43 & $X_{17} \subset \mathbb{P}(1,3,7,8)$ & 2 & $17 / 42$ & 1 & 11 & 9 & $\frac{1}{3}(1,1), \frac{1}{7}(1,1), \frac{1}{8}(1,5)$ \\
\hline 44 & $X_{17} \subset \mathbb{P}\left(1^{2}, 7,10\right)$ & 2 & $34 / 35$ & 3 & 19 & 17 & $\frac{1}{7}(1,1), \frac{1}{10}(1,1)$ \\
\hline 45 & $X_{17} \subset \mathbb{P}\left(1^{2}, 8,9\right)$ & 2 & $17 / 18$ & 3 & 19 & 17 & $\frac{1}{8}(1,1), \frac{1}{9}(1,1)$ \\
\hline 46 & $X_{18} \subset \mathbb{P}\left(1^{2}, 8,10\right)$ & 2 & $9 / 10$ & 3 & 20 & 18 & $\frac{1}{8}(1,1), \frac{1}{10}(1,1)$ \\
\hline 47 & $X_{18} \subset \mathbb{P}\left(1^{2}, 9^{2}\right)$ & 2 & $8 / 9$ & 3 & 20 & 18 & $2 \times \frac{1}{9}(1,1)$ \\
\hline 48 & $X_{19} \subset \mathbb{P}\left(1^{2}, 9,10\right)$ & 2 & $38 / 45$ & 3 & 21 & 19 & $\frac{1}{9}(1,1), \frac{1}{10}(1,1)$ \\
\hline 49 & $X_{20} \subset \mathbb{P}\left(1^{2}, 10^{2}\right)$ & 2 & $4 / 5$ & 3 & 22 & 20 & $2 \times \frac{1}{10}(1,1)$ \\
\hline 50 & $X_{21} \subset \mathbb{P}\left(3,6,7^{2}\right)$ & 2 & $2 / 21$ & 0 & 9 & 7 & $3 \times \frac{1}{3}(1,1), \frac{1}{6}(1,1), 3 \times \frac{1}{7}(1,2)$ \\
\hline 51 & $X_{21} \subset \mathbb{P}(1,3,9,10)$ & 2 & $14 / 45$ & 1 & 13 & 11 & $\left.2 \times \frac{1}{3}(1,1), \frac{1}{9}(1,1), \frac{1}{10}(1,3)\right)$ \\
\hline 52 & $X_{22} \subset \mathbb{P}(1,5,7,11)$ & 2 & $8 / 35$ & 1 & 10 & 8 & $\frac{1}{5}(1,1), \frac{1}{7}(1,3)$ \\
\hline 53 & $X_{24} \subset \mathbb{P}\left(3,7,8^{2}\right)$ & 2 & $1 / 14$ & 0 & 7 & 5 & $\frac{1}{7}(1,1), 3 \times \frac{1}{8}(1,5)$ \\
\hline 54 & $X_{30} \subset \mathbb{P}\left(3,9,10^{2}\right)$ & 2 & $2 / 45$ & 0 & 9 & 7 & $\left.3 \times \frac{1}{3}(1,1), \frac{1}{9}(1,1), 3 \times \frac{1}{10}(1,3)\right)$ \\
\hline 55 & $X_{36} \subset \mathbb{P}(1,7,12,18)$ & 2 & $2 / 21$ & 1 & 11 & 9 & $\frac{1}{6}(1,1), \frac{1}{7}(1,3)$ \\
\hline 56 & $X_{6} \subset \mathbb{P}\left(1^{2}, 2,5\right)$ & 3 & $27 / 5$ & 6 & 5 & 3 & $\frac{1}{5}(1,2)$ \\
\hline 57 & $X_{8} \subset \mathbb{P}\left(1^{2}, 2,7\right)$ & 3 & $36 / 7$ & 6 & 6 & 4 & $\frac{1}{7}(1,2)$ \\
\hline 58 & $X_{10} \subset \mathbb{P}\left(1,2,5^{2}\right)$ & 3 & $9 / 5$ & 2 & 7 & 5 & $2 \times \frac{1}{5}(1,2)$ \\
\hline 59 & $X_{12} \subset \mathbb{P}(1,2,5,7)$ & 3 & $54 / 35$ & 2 & 8 & 6 & $\frac{1}{5}(1,2), \frac{1}{7}(1,2)$ \\
\hline 60 & $X_{14} \subset \mathbb{P}\left(1,2,7^{2}\right)$ & 3 & $9 / 7$ & 2 & 9 & 7 & $2 \times \frac{1}{7}(1,2)$ \\
\hline 61 & $X_{15} \subset \mathbb{P}\left(1,5^{2}, 7\right)$ & 3 & $27 / 35$ & 1 & 9 & 3 & $3 \times \frac{1}{5}(1,2), \frac{1}{7}(1,1)$ \\
\hline 62 & $X_{6} \subset \mathbb{P}\left(1^{2}, 3,5\right)$ & 4 & $32 / 5$ & 7 & 4 & 2 & $\frac{1}{5}(1,2)$ \\
\hline 63 & $X_{10} \subset \mathbb{P}\left(1,3,5^{2}\right)$ & 4 & $32 / 15$ & 2 & 6 & 4 & $\frac{1}{3}(1,1), 2 \times \frac{1}{5}(1,2)$ \\
\hline 64 & $X_{12} \subset \mathbb{P}(1,3,5,7)$ & 4 & $64 / 35$ & 2 & 6 & 4 & $\frac{1}{5}(1,2), \frac{1}{7}(1,3)$ \\
\hline 65 & $X_{15} \subset \mathbb{P}(1,3,5,10)$ & 4 & $8 / 5$ & 2 & 7 & 5 & $\frac{1}{5}(1,2), \frac{1}{10}(1,3)$ \\
\hline 66 & $X_{15} \subset \mathbb{P}\left(3,5^{2}, 6\right)$ & 4 & $8 / 15$ & 0 & 7 & 5 & $2 \times \frac{1}{3}(1,1), 3 \times \frac{1}{5}(1,2), \frac{1}{6}(1,1)$ \\
\hline 67 & $X_{21} \subset \mathbb{P}\left(1,7^{2}, 10\right)$ & 4 & $24 / 35$ & 1 & 9 & 7 & $3 \times \frac{1}{7}(1,3), \frac{1}{10}(1,1)$ \\
\hline 68 & $X_{8} \subset \mathbb{P}(1,2,3,7)$ & 5 & $100 / 21$ & 5 & 4 & 2 & $\frac{1}{3}(1,1), \frac{1}{7}(1,3)$ \\
\hline 69 & $X_{8} \subset \mathbb{P}\left(1^{2}, 4,7\right)$ & 5 & $50 / 7$ & 8 & 4 & 2 & $\frac{1}{7}(1,2)$ \\
\hline 70 & $X_{12} \subset \mathbb{P}(1,3,4,9)$ & 5 & $25 / 9$ & 3 & 5 & 3 & $\frac{1}{3}(1,1), \frac{1}{9}(1,4)$ \\
\hline 71 & $X_{14} \subset \mathbb{P}\left(2,3,7^{2}\right)$ & 5 & $25 / 21$ & 1 & 5 & 3 & $\frac{1}{3}(1,1), 2 \times \frac{1}{7}(1,3)$ \\
\hline 72 & $X_{16} \subset \mathbb{P}(1,4,7,9)$ & 5 & $100 / 63$ & 2 & 6 & 4 & $\frac{1}{7}(1,2), \frac{1}{9}(1,4)$ \\
\hline
\end{tabular}


Table A1. Cont.

\begin{tabular}{cccccccc}
\hline S.No & $\boldsymbol{X}$ & $\boldsymbol{I}$ & $-\boldsymbol{K}_{\boldsymbol{X}}^{2}$ & $\boldsymbol{h}^{0}\left(-\boldsymbol{K}_{\boldsymbol{X}}\right)$ & $\boldsymbol{e}(\boldsymbol{X})$ & $\boldsymbol{\rho}(\boldsymbol{X})$ & Basket $\mathcal{B}$ \\
\hline 73 & $X_{21} \subset \mathbb{P}\left(3,7^{2}, 9\right)$ & 5 & $25 / 63$ & 0 & 7 & 5 & $2 \times \frac{1}{3}(1,1), 3 \times \frac{1}{7}(1,3), \frac{1}{9}(1,1)$ \\
\hline 74 & $X_{15} \subset \mathbb{P}(1,5,7,8)$ & 6 & $27 / 14$ & 2 & 5 & 3 & $\frac{1}{7}(1,3), \frac{1}{8}(1,5)$ \\
75 & $X_{16} \subset \mathbb{P}\left(1,5,8^{2}\right)$ & 6 & $9 / 5$ & 2 & 6 & 4 & $\frac{1}{5}(1,1), 2 \times \frac{1}{8}(1,5)$ \\
\hline 76 & $X_{10} \subset \mathbb{P}(1,2,5,9)$ & 7 & $49 / 9$ & 6 & 3 & 1 & $\frac{1}{9}(1,4)$ \\
77 & $X_{12} \subset \mathbb{P}(2,3,5,9)$ & 7 & $98 / 45$ & 2 & 4 & 2 & $\frac{1}{3}(1,1), \frac{1}{5}(1,2), \frac{1}{9}(1,4)$ \\
\hline 78 & $X_{8} \subset \mathbb{P}(1,3,5,7)$ & 8 & $512 / 105$ & 5 & 4 & 2 & $\frac{1}{3}(1,1), \frac{1}{5}(1,2), \frac{1}{7}(1,2)$ \\
79 & $X_{14} \subset \mathbb{P}(1,5,7,9)$ & 8 & $128 / 45$ & 3 & 4 & 2 & $\frac{1}{5}(1,2), \frac{1}{9}(1,4)$ \\
80 & $X_{15} \subset \mathbb{P}(1,6,7,9)$ & 8 & $160 / 63$ & 3 & 5 & 3 & $\frac{1}{6}(1,1), \frac{1}{7}(1,3), \frac{1}{9}(1,4)$ \\
81 & $X_{15} \subset \mathbb{P}(1,5,7,10)$ & 8 & $96 / 35$ & 3 & 5 & 3 & $\left.\frac{1}{5}(1,2), \frac{1}{7}(1,2), \frac{1}{10}(1,3)\right)$ \\
\hline
\end{tabular}

Table A2. Codimension 2 Complete Intersections.

\begin{tabular}{|c|c|c|c|c|c|c|c|}
\hline S.No & $X$ & $I$ & $-K_{X}^{2}$ & $h^{0}\left(-K_{X}\right)$ & $e(X)$ & $\rho(X)$ & Basket $\mathcal{B}$ \\
\hline 82 & $X_{2,2} \subset \mathbb{P}\left(1^{5}\right)$ & 1 & 4 & 5 & 8 & 8 & \\
\hline 83 & $X_{4^{2}} \subset \mathbb{P}\left(1^{2}, 2^{2}, 3\right)$ & 1 & $4 / 3$ & 2 & 10 & $28 / 3$ & $\frac{1}{3}(1,1)[6]$ \\
\hline 84 & $X_{4,6} \subset \mathbb{P}\left(1,2^{2}, 3^{2}\right)$ & 1 & $2 / 3$ & 1 & 10 & $26 / 3$ & $2 \times \frac{1}{3}(1,1)[6]$ \\
\hline 85 & $X_{6^{2}} \subset \mathbb{P}\left(1^{2}, 3^{2}, 5\right)$ & 1 & $4 / 5$ & 2 & 12 & $56 / 5$ & $\frac{1}{5}(1,1)[8]$ \\
\hline 86 & $X_{6^{2}} \subset \mathbb{P}\left(2^{2}, 3^{3}\right)$ & 1 & $1 / 3$ & 0 & 9 & $19 / 3$ & $4 \times \frac{1}{3}(1,1)[6]$ \\
\hline 87 & $X_{6,7} \subset \mathbb{P}\left(1,2,3^{2}, 5\right)$ & 1 & $7 / 15$ & 1 & 11 & $133 / 15$ & $2 \times \frac{1}{3}(1,1), \frac{1}{5}(1,1)$ \\
\hline 88 & $X_{6,8} \subset \mathbb{P}(1,2,3,4,5)$ & 1 & $2 / 5$ & 1 & 10 & $46 / 5$ & $\frac{1}{5}(1,2)$ \\
\hline 89 & $X_{8^{2}} \subset \mathbb{P}\left(1^{2}, 4^{2}, 7\right)$ & 1 & $4 / 7$ & 2 & 14 & $92 / 7$ & $\frac{1}{7}(1,1)$ \\
\hline 90 & $X_{6,10} \subset \mathbb{P}\left(1,3^{2}, 5^{2}\right)$ & 1 & $4 / 15$ & 1 & 12 & $136 / 15$ & $2 \times \frac{1}{3}(1,1), 2 \times \frac{1}{5}(1,1)$ \\
\hline 91 & $X_{8,10} \subset \mathbb{P}\left(2,3,4,5^{2}\right)$ & 1 & $2 / 15$ & 0 & 8 & $86 / 15$ & $\frac{1}{3}(1,1), 2 \times \frac{1}{5}(1,2)$ \\
\hline 92 & $X_{9,10} \subset \mathbb{P}\left(2,3^{2}, 5,7\right)$ & 1 & $1 / 7$ & 0 & 9 & $43 / 7$ & $3 \times \frac{1}{3}(1,1), \frac{1}{7}(1,2)$ \\
\hline 93 & $X_{10^{2}} \subset \mathbb{P}\left(1^{2}, 5^{2}, 9\right)$ & 1 & $4 / 9$ & 2 & 16 & $136 / 9$ & $\frac{1}{9}(1,1)$ \\
\hline 94 & $X_{10,11} \subset \mathbb{P}\left(1,2,5^{2}, 9\right)$ & 1 & $11 / 45$ & 1 & 13 & $473 / 45$ & $2 \times \frac{1}{5}(1,2), \frac{1}{9}(1,1)$ \\
\hline 95 & $X_{10,12} \subset \mathbb{P}\left(3^{2}, 5^{2}, 7\right)$ & 1 & $8 / 105$ & 0 & 10 & $512 / 105$ & $4 \times \frac{1}{3}(1,1), 2 \times \frac{1}{5}(1,1), \frac{1}{7}(1,2)$ \\
\hline 96 & $X_{10,12} \subset \mathbb{P}(2,3,5,6,7)$ & 1 & $2 / 21$ & 0 & 8 & $122 / 21$ & $2 \times \frac{1}{3}(1,1), \frac{1}{7}(1,3)$ \\
\hline 97 & $X_{6,8} \subset \mathbb{P}\left(1,3^{2}, 4,5\right)$ & 2 & $16 / 15$ & 1 & 8 & $88 / 15$ & $2 \times \frac{1}{3}(1,1), \frac{1}{5}(1,2)$ \\
\hline 98 & $X_{8,10} \subset \mathbb{P}(1,3,4,5,7)$ & 2 & $16 / 21$ & 1 & 8 & $136 / 21$ & $\frac{1}{3}(1,1), \frac{1}{7}(1,3)$ \\
\hline 99 & $X_{8,12} \subset \mathbb{P}(1,3,5,6,7)$ & 2 & $64 / 105$ & 1 & 10 & $736 / 105$ & $2 \times \frac{1}{3}(1,1), \frac{1}{5}(1,1), \frac{1}{7}(1,2)$ \\
\hline 100 & $X_{10,12} \subset \mathbb{P}\left(3,4,5^{2}, 7\right)$ & 2 & $8 / 35$ & 0 & 6 & $124 / 35$ & $2 \times \frac{1}{5}(1,2), \frac{1}{7}(1,3)$ \\
\hline 101 & $X_{9,14} \subset \mathbb{P}(1,3,6,7,8)$ & 2 & $1 / 2$ & 1 & 10 & $61 / 8$ & $\frac{1}{3}(1,1), \frac{1}{6}(1,1), \frac{1}{8}(1,5)$ \\
\hline 102 & $X_{12,14} \subset \mathbb{P}(3,4,5,7,9)$ & 2 & $8 / 45$ & 0 & 6 & $164 / 45$ & $\frac{1}{3}(1,1), \frac{1}{5}(1,2), \frac{1}{9}(1,4)$ \\
\hline 103 & $X_{14,15} \subset \mathbb{P}\left(3,6,7^{2}, 8\right)$ & 2 & $5 / 42$ & 0 & 8 & $545 / 168$ & $2 \times \frac{1}{3}(1,1), \frac{1}{6}(1,1), 2 \times \frac{1}{7}(1,2), \frac{1}{8}(1,5)$ \\
\hline 104 & $X_{11,18} \subset \mathbb{P}(1,3,8,9,10)$ & 2 & $11 / 30$ & 1 & 12 & $1067 / 120$ & $2 \times \frac{1}{3}(1,1), \frac{1}{8}(1,1), \frac{1}{10}(1,3)$ \\
\hline 105 & $X_{12,14} \subset \mathbb{P}\left(4,5,6,7^{2}\right)$ & 3 & $9 / 35$ & 0 & 5 & $87 / 35$ & $\frac{1}{5}(1,2), 2 \times \frac{1}{7}(1,3)$ \\
\hline 106 & $X_{10,12} \subset \mathbb{P}\left(3,5^{2}, 6,7\right)$ & 4 & $64 / 105$ & 0 & 6 & $232 / 105$ & $2 \times \frac{1}{3}(1,1), 2 \times \frac{1}{5}(1,2), \frac{1}{7}(1,2)$ \\
\hline
\end{tabular}


Table A3. Codimension 3 Pfaffians.

\begin{tabular}{|c|c|c|c|c|c|c|c|c|c|}
\hline S.No & $X$ & $I$ & $-K^{2}$ & $h^{0}(-K)$ & Basket $\mathcal{B}$ & \multicolumn{4}{|c|}{ Weight Matrix } \\
\hline 107 & $\begin{array}{l}X_{2,2,2,2,2} \\
\quad \subset \mathbb{P}\left(1^{6}\right)\end{array}$ & 1 & 5 & 6 & & 1 & $\begin{array}{l}1 \\
1\end{array}$ & $\begin{array}{l}1 \\
1 \\
1\end{array}$ & $\begin{array}{l}1 \\
1 \\
1 \\
1\end{array}$ \\
\hline 108 & $\begin{array}{l}X_{3,3,4,4,4} \\
\quad \subset \mathbb{P}\left(1^{3}, 2^{2}, 3\right)\end{array}$ & 1 & $7 / 3$ & 3 & $\frac{1}{3}(1,1)[6]$ & 1 & $\begin{array}{l}1 \\
1\end{array}$ & $\begin{array}{l}2 \\
2 \\
2\end{array}$ & $\begin{array}{l}2 \\
2 \\
2 \\
3\end{array}$ \\
\hline 109 & $\begin{array}{l}X_{4,4,6,6,6} \\
\quad \subset \mathbb{P}\left(1^{3}, 3^{2}, 5\right)\end{array}$ & 1 & $9 / 5$ & 3 & $\frac{1}{5}(1,1)[8]$ & 1 & $\begin{array}{l}1 \\
1\end{array}$ & $\begin{array}{l}3 \\
3 \\
3\end{array}$ & $\begin{array}{l}3 \\
3 \\
3 \\
5\end{array}$ \\
\hline 110 & $\begin{array}{l}X_{4,5,6,6,7} \\
\quad \subset \mathbb{P}\left(1^{2}, 2,3^{2}, 5\right)\end{array}$ & 1 & $17 / 15$ & 2 & $\frac{1}{3}(1,1), \frac{1}{5}(1,1)$ & 1 & $\begin{array}{l}1 \\
2\end{array}$ & $\begin{array}{l}2 \\
3 \\
3\end{array}$ & $\begin{array}{l}3 \\
4 \\
4 \\
5\end{array}$ \\
\hline 111 & $\begin{array}{l}X_{5,5,8,8,8} \\
\quad \subset \mathbb{P}\left(1^{3}, 4^{2}, 7\right)\end{array}$ & 1 & $11 / 7$ & 3 & $\frac{1}{7}(1,1)$ & 1 & $\begin{array}{l}1 \\
1\end{array}$ & $\begin{array}{l}4 \\
4 \\
4\end{array}$ & $\begin{array}{l}4 \\
4 \\
4 \\
7\end{array}$ \\
\hline 112 & $\begin{array}{l}X_{6,7,8,9,10} \\
\quad \subset \mathbb{P}\left(1,2,3^{2}, 5,7\right)\end{array}$ & 1 & $10 / 21$ & 1 & $\frac{1}{3}(1,1), \frac{1}{7}(1,4)$ & 1 & $\begin{array}{l}2 \\
3\end{array}$ & $\begin{array}{l}3 \\
4 \\
5\end{array}$ & $\begin{array}{l}4 \\
5 \\
6 \\
7\end{array}$ \\
\hline 113 & $\begin{array}{l}X_{6,6,10,10,10} \\
\quad \subset \mathbb{P}\left(1^{3}, 5^{2}, 9\right)\end{array}$ & 1 & $13 / 9$ & 3 & $\frac{1}{9}(1,1)$ & 1 & $\begin{array}{l}1 \\
1\end{array}$ & $\begin{array}{l}5 \\
5 \\
5\end{array}$ & $\begin{array}{l}5 \\
5 \\
5 \\
9\end{array}$ \\
\hline 114 & $\begin{array}{l}X_{7,8,8,9,10} \\
\quad \subset \mathbb{P}\left(2,3^{2}, 4,5^{2}\right)\end{array}$ & 1 & $1 / 5$ & 0 & $3 \times \frac{1}{3}(1,1), \frac{1}{5}(1,2), \frac{1}{5}(1,1)$ & 2 & $\begin{array}{l}3 \\
4\end{array}$ & $\begin{array}{l}3 \\
4 \\
5\end{array}$ & $\begin{array}{l}4 \\
5 \\
6 \\
6\end{array}$ \\
\hline 115 & $\begin{array}{l}X_{6,7,10,10,11} \\
\quad \subset \mathbb{P}\left(1^{2}, 2,5^{2}, 9\right)\end{array}$ & 1 & $38 / 45$ & 2 & $\frac{1}{5}(1,2), \frac{1}{9}(1,1)$ & 1 & $\begin{array}{l}1 \\
2\end{array}$ & $\begin{array}{l}4 \\
5 \\
5\end{array}$ & $\begin{array}{l}5 \\
6 \\
6 \\
9\end{array}$ \\
\hline 116 & $\begin{array}{l}X_{6,8,10,10,12} \\
\quad \subset \mathbb{P}\left(1,3^{2}, 5^{2}, 7\right)\end{array}$ & 1 & $29 / 105$ & 1 & $\frac{1}{3}(1,1), \frac{1}{5}(1,1), \frac{1}{7}(1,4)$ & 1 & $\begin{array}{l}1 \\
3\end{array}$ & $\begin{array}{l}3 \\
5 \\
5\end{array}$ & $\begin{array}{l}5 \\
7 \\
7 \\
9\end{array}$ \\
\hline 117 & $\begin{array}{l}X_{10,10,12,12,14} \\
\quad \subset \mathbb{P}\left(3^{2}, 5^{2}, 7^{2}\right)\end{array}$ & 1 & $3 / 35$ & 0 & $3 \times \frac{1}{3}(1,1), \frac{1}{5}(1,1), 2 \times \frac{1}{7}(1,4)$ & 3 & $\begin{array}{l}3 \\
5\end{array}$ & $\begin{array}{l}5 \\
7 \\
7\end{array}$ & $\begin{array}{l}5 \\
7 \\
7 \\
9\end{array}$ \\
\hline
\end{tabular}


Table A3. Cont.

\begin{tabular}{|c|c|c|c|c|c|c|c|c|c|}
\hline S.No & $x$ & $I$ & $-K^{2}$ & $h^{0}(-K)$ & Basket $\mathcal{B}$ & \multicolumn{4}{|c|}{ Weight Matri } \\
\hline 118 & $\begin{array}{l}X_{11,12,12,15,16} \\
\quad \subset \mathbb{P}\left(2,5^{2}, 6,7,9\right)\end{array}$ & 1 & $23 / 315$ & 0 & $3 \times \frac{1}{5}(1,2), \frac{1}{7}(1,3), \frac{1}{9}(1,1)$ & & $\begin{array}{l}5 \\
6\end{array}$ & $\begin{array}{l}5 \\
6 \\
9\end{array}$ & $\begin{array}{c}6 \\
7 \\
10 \\
10\end{array}$ \\
\hline 119 & $\begin{array}{l}X_{4,7,8,8,9} \\
\quad \subset \mathbb{P}\left(1^{2}, 2,3,6,7\right)\end{array}$ & 2 & $22 / 7$ & 4 & $\frac{1}{3}(1,1), \frac{1}{6}(1,1), \frac{1}{7}(1,2)$ & 1 & & $\begin{array}{l}2 \\
3 \\
3\end{array}$ & $\begin{array}{l}5 \\
6 \\
6 \\
7\end{array}$ \\
\hline 120 & $\begin{array}{l}X_{4,8,9,9,10} \\
\quad \subset \mathbb{P}\left(1^{2}, 2,3,7,8\right)\end{array}$ & 2 & $43 / 14$ & 4 & $\frac{1}{7}(1,1), \frac{1}{8}(1,5)$ & 1 & $\begin{array}{l}1 \\
2\end{array}$ & $\begin{array}{l}2 \\
3 \\
3\end{array}$ & $\begin{array}{l}6 \\
7 \\
7 \\
8\end{array}$ \\
\hline 121 & $\begin{array}{l}X_{4,10,11,11,12} \\
\quad \subset \mathbb{P}\left(1^{2}, 2,3,9,10\right)\end{array}$ & 2 & $134 / 45$ & 4 & $\frac{1}{3}(1,1), \frac{1}{9}(1,1), \frac{1}{10}(1,3)$ & 1 & $\begin{array}{l}1 \\
2\end{array}$ & $\begin{array}{l}2 \\
3 \\
3\end{array}$ & $\begin{array}{c}8 \\
9 \\
9 \\
10\end{array}$ \\
\hline 122 & $\begin{array}{l}X_{8,9,12,13,14} \\
\quad \subset \mathbb{P}(1,3,5,6,7,8)\end{array}$ & 2 & $19 / 30$ & 1 & $\frac{1}{3}(1,1), \frac{1}{5}(1,1), \frac{1}{8}(1,5)$ & 1 & $\begin{array}{l}2 \\
3\end{array}$ & $\begin{array}{l}5 \\
6 \\
7\end{array}$ & $\begin{array}{c}6 \\
7 \\
8 \\
11\end{array}$ \\
\hline 123 & $\begin{array}{l}X_{12,12,14,15,15} \\
\quad \subset \mathbb{P}\left(4,5^{2}, 7^{2}, 8\right)\end{array}$ & 2 & $11 / 70$ & 0 & $2 \times \frac{1}{5}(1,2), 2 \times \frac{1}{7}(1,3), \frac{1}{8}(1,1)$ & & $\begin{array}{l}5 \\
5\end{array}$ & $\begin{array}{l}7 \\
7 \\
8\end{array}$ & $\begin{array}{c}7 \\
7 \\
8 \\
10\end{array}$ \\
\hline 124 & $\begin{array}{l}X_{14,14,15,15,16} \\
\quad \subset \mathbb{P}\left(3,6,7^{2}, 8^{2}\right)\end{array}$ & 2 & $1 / 7$ & 0 & $\frac{1}{3}(1,1), \frac{1}{6}(1,1), \frac{1}{7}(1,2), 2 \times \frac{1}{8}(1,5)$ & 6 & $\begin{array}{l}6 \\
7\end{array}$ & $\begin{array}{l}7 \\
8 \\
8\end{array}$ & $\begin{array}{l}7 \\
8 \\
8 \\
9\end{array}$ \\
\hline 125 & $\begin{array}{l}X_{16,17,17,18,18} \\
\quad \subset \mathbb{P}\left(3,7,8^{2}, 9,10\right)\end{array}$ & 2 & $11 / 105$ & 0 & $\frac{1}{3}(1,1), \frac{1}{7}(1,1), 2 \times \frac{1}{8}(1,5), \frac{1}{10}(1,3)$ & 7 & $\begin{array}{l}8 \\
8\end{array}$ & $\begin{array}{l}8 \\
8 \\
9\end{array}$ & $\begin{array}{c}9 \\
9 \\
10 \\
10\end{array}$ \\
\hline 126 & $\begin{array}{l}X_{6 \cdot 6,8,8,10} \\
\quad \subset \mathbb{P}\left(1^{2}, 3,5^{2}, 7\right)\end{array}$ & 3 & $153 / 35$ & 4 & $2 \times \frac{1}{5}(1,2), \frac{1}{7}(1,1)$ & 1 & $\begin{array}{l}1 \\
3\end{array}$ & $\begin{array}{l}3 \\
5 \\
5\end{array}$ & $\begin{array}{l}3 \\
5 \\
5 \\
7\end{array}$ \\
\hline 127 & $\begin{array}{l}X_{8,8,11,11,14} \\
\quad \subset \mathbb{P}\left(1^{2}, 4,7^{2}, 10\right)\end{array}$ & 4 & $184 / 35$ & 6 & $2 \times \frac{1}{7}(1,3), \frac{1}{10}(1,1)$ & 1 & $\begin{array}{l}1 \\
4\end{array}$ & $\begin{array}{l}4 \\
7 \\
7\end{array}$ & $\begin{array}{c}4 \\
7 \\
7 \\
10\end{array}$ \\
\hline
\end{tabular}


Table A4. Codimension $4 \mathbb{P}^{2} \times \mathbb{P}^{2}$.

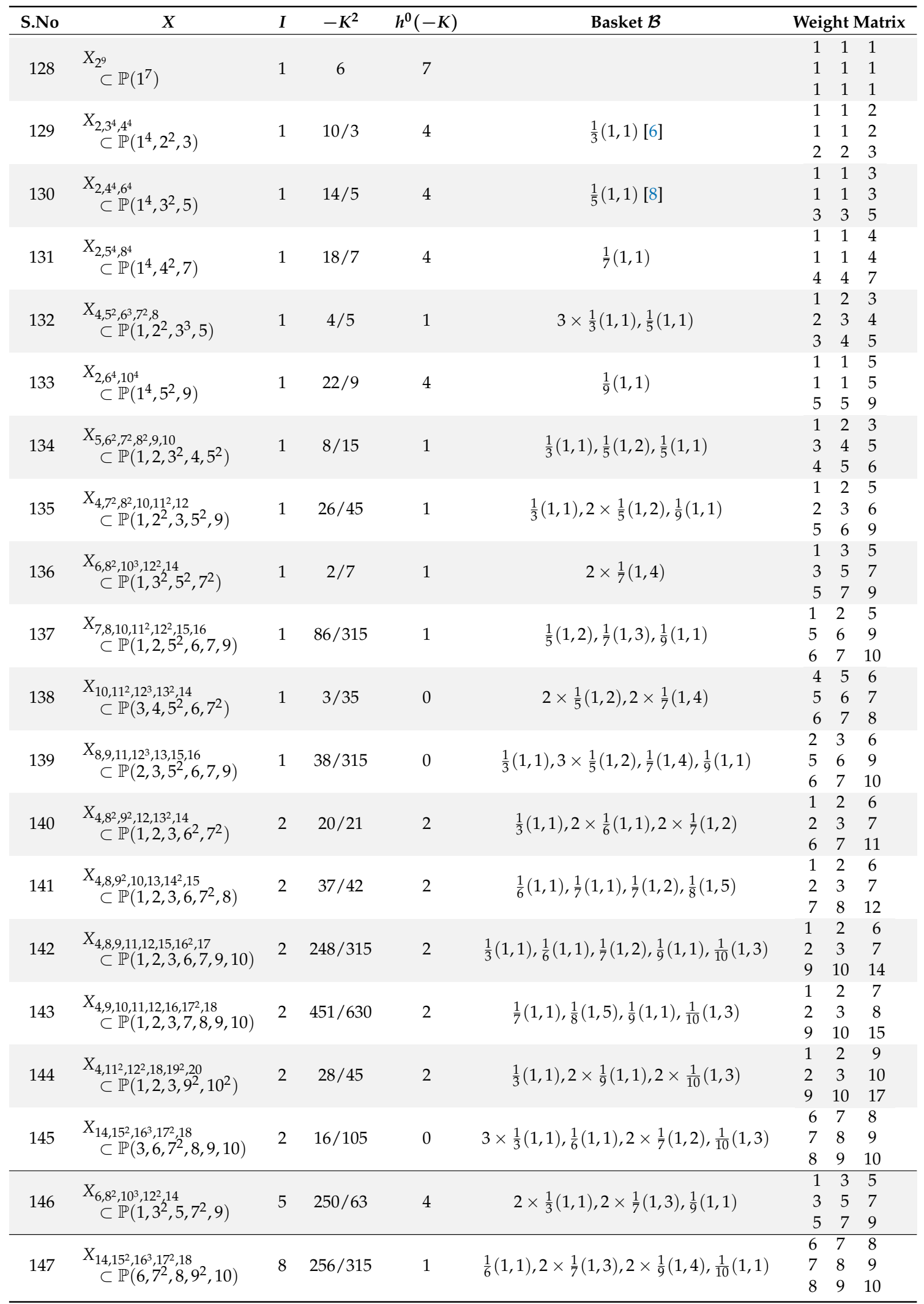




\section{References}

1. Cheltsov, I.; Park, J.; Shramov, C. Exceptional Del Pezzo hypersurfaces. J. Geom. Anal. 2010, 20, 787-816. [CrossRef]

2. Cheltsov, I.; Shramov, C. Del Pezzo zoo. Exp. Math. 2013, 22, 313-326. [CrossRef]

3. Coates, T.; Corti, A.; Galkin, S.; Golyshev, V.; Kasprzyk, A. Mirror symmetry and Fano manifolds. In Proceedings of the European Congress of Mathematics, Krakow, Poland, 2-7 July 2012; European Mathematical Society Publishing House: Zurich, Switzerland, 2012, 824p; ISBN 978-3-03719-120. [CrossRef]

4. Akhtar, M.; Coates, T.; Corti, A.; Heuberger, L.; Kasprzyk, A.; Oneto, A.; Petracci, A.; Prince, T.; Tveiten, K. Mirror symmetry and the classification of orbifold Del Pezzo surfaces. Proc. Am. Math. Soc. 2016, 144, 513-527. [CrossRef]

5. Kasprzyk, A.; Nill, B.; Prince, T. Minimality and mutation-equivalence of polygons. In Forum of Mathematics, Sigma; Cambridge University Press: Cambridge, UK, 2017; Volume 5.

6. Corti, A.; Heuberger, L. Del Pezzo surfaces with $\frac{1}{3}(1,1)$ points. Manuscripta Math. 2017, 153, 71-118. [CrossRef]

7. Cavey, D.; Prince, T. Del Pezzo surfaces with a single $\frac{1}{k}(1,1)$ singularity. J. Math. Soc. Jpn. 2020, 72, 465-505. [CrossRef]

8. Cavey, D.; Kutas, E. Classification of Minimal Polygons with Specified Singularity Content. arXiv 2017, arXiv:1703.05266.

9. Miura, T. Classification of del Pezzo surfaces with $\frac{1}{3}(1,1)$ and $\frac{1}{4}(1,1)$ singularities. arXiv 2019, arXiv:1903.00679.

10. Reid, M.; Suzuki, K. Cascades of projections from log del Pezzo surfaces. In Number Theory and Algebraic Geometry; London Mathematical Society Lecture Note Series; Reid, M., Skorobogatov, A., Eds.; Cambridge University Press: Cambridge, UK, 2004; pp. 227-250. [CrossRef]

11. Corti, A.; Reid, M. Weighted Grassmannians. In Algebraic Geometry; Beltrametti, M.C., Catanese, F., Ciliberto, C., Lanteri, A., Pedrini, C., Eds.; de Gruyter: Berlin, Germany, 2002; pp. 141-163.

12. Qureshi, M.I.; Szendrői, B. Constructing projective varieties in weighted flag varieties. Bull. Lond. Math. Soc. 2011, 43, 786-798. [CrossRef]

13. Qureshi, M.I.; Szendrői, B. Calabi-Yau threefolds in weighted flag varieties. Adv. High Energy Phys. 2012, 2012, 547317. [CrossRef]

14. Brown, G.; Kasprzyk, A.M.; Qureshi, M.I. Fano 3-folds in $\mathbb{P}^{2} \times \mathbb{P}^{2}$ format, Tom and Jerry. Eur. J. Math. 2018, 4, 51-72. [CrossRef]

15. Brown, G.; Kasprzyk, A.M.; Zhu, L. Gorenstein formats, canonical and Calabi-Yau threefolds. Exp. Math. 2019, 1-19. [CrossRef]

16. Qureshi, M.I. Computing isolated orbifolds in weighted flag varieties. J. Symb. Comput. 2017, 79 Pt 2, 457-474. [CrossRef]

17. Mayanskiy, E. Weighted complete intersection del Pezzo surfaces. arXiv 2016, arXiv:1608.02049.

18. Paemurru, E. Del Pezzo surfaces in weighted projective spaces. Proc. Edinb. Math. Soc. 2018, 61, 545-572. [CrossRef]

19. Qureshi, M.I. Biregular models of log del Pezzo surfaces with rigid singularities. Math. Comput. 2019, 88, 2497-2521. [CrossRef]

20. Atiyah, M.F.; Macdonald, I.G. Introduction to Commutative Algebra; Reading, Mass.-London-Don Mills, Ont.; Addison-Wesley Publishing Co.: Boston, MA, USA, 1969; p. ix+128.

21. Kollár, J.; Shepherd-Barron, N.I. Threefolds and deformations of surface singularities. Invent. Math. 1988, 91, 299-338.

22. Akhtar, M.; Kasprzyk, A. Singularity content. arXiv 2014, arXiv:1401.5458.

23. Szendrői, B. On Weighted Homogeneous Varieties; 2005. Unpublished manuscript.

24. Buckley, A.; Reid, M.; Zhou, S. Ice cream and orbifold Riemann-Roch. Izv. Math. 2013, 77, 461-486. [CrossRef]

25. Blache, R. Chern classes and Hirzebruch-Riemann-Roch theorem for coherent sheaves on complex-projective orbifolds with isolated singularities. Math. Z. 1996, 222, 7-57. [CrossRef]

26. Brown, G.; Fatighenti, E. Hodge numbers and deformations of Fano 3-folds. arXiv 2017, arXiv:1707.00653. 
27. Dolgachev, I. Weighted projective spaces. In Group Actions and Vector Fields; Lec Note in Mathematics; Springer: Berlin/Heidelberg, Germany, 1981; Volume 956, pp. 34-71.

28. Hwang, D. On the orbifold Euler characteristic of log del Pezzo surfaces of rank one. J. Korean Math. Soc. 2014, 51, 867-879. [CrossRef]

29. Iano-Fletcher, A.R. Working with weighted complete intersections. In Explicit Birational Geometry of 3-Folds; London Math. Soc. Lecture Note Ser; CUP: London, UK, 2000; Volume 281, pp. 101-173.

30. Bosma, W.; Cannon, J.; Playoust, C. The Magma algebra system. I. The user language. J. Symb. Comput. 1997, 24, 235-265. Computational algebra and number theory (London, 1993). [CrossRef]

31. Brown, G.; Zucconi, F. Graded rings of rank 2 Sarkisov links. Nagoya Math. J. 2010, 197, 1-44. [CrossRef]

(C) 2020 by the author. Licensee MDPI, Basel, Switzerland. This article is an open access article distributed under the terms and conditions of the Creative Commons Attribution (CC BY) license (http://creativecommons.org/licenses/by/4.0/). 